\title{
Effect of Substrate Layer Thickness on the AC Losses in Stacked Superconducting Pancake Coils using Direct H-formulations
}

\author{
Abhinav Kumar, Ashish Agrawal
}

\begin{abstract}
Advanced electric aircrafts are in their design phase and superconducting machines are going to be the part of such fascinating technology. In order to diminish the losses involved due to conventional copper conductors, superconductors are proposed for the electric aircraft applications by the American research agencies like NASA and AFRL.

Usually, Pancake coils are frequently used in various electric aircraft power applications including high speed motors, generators, transformers and solenoid magnets. Coils are generally bound with high temperature superconducting (HTS) tapes like BSCCO and YBCO. Mostly, $2^{\text {nd }}$ generation coated conductors (YBCO) are employed in power applications due to their merits over BSCCO ( $1^{\text {st }}$ generation tapes). A superconducting tape manufactured by SuperPower through iBAD manufacturing technique generally consist copper stabilizer, silver over-layer, YBCO layer, buffer layer, substrate material followed by copper stabilizer. The volume fraction of the substrate material and copper stabilizer is more than $90 \%$ in the proposed tape. In the present work, the thickness of the substrate material has been varied to evaluate the AC losses involved in the above mentioned applications due to time-varying magnetic fields. A current of 270 A $\left(I_{c}=330 A\right)$ is flowing through a coil of 108 turns. AC loss has been evaluated for various thicknesses $30 \mu \mathrm{m}$ to $90 \mu \mathrm{m}$ at a frequency of $50 \mathrm{~Hz}$. The simulations are done using COMSOL MultiPhysics ${ }^{\circledR}$ commercial software package.
\end{abstract}

Keywords : Substrate Layer, Superconductors, Pancake coils, H-formulations, YBCO, iBAD manufacturing technique.

\section{INTRODUCTION}

Electric aircrafts are receiving great attention in the field of aviation from last decade and research communities are doing their best to identify the best possible machines through which highly efficient aircrafts may come into picture. The need of electric aircrafts is generated due to the depletion of fossil fuels used in the field of aviation and the challenges associated with the air pollution. For the instant relief, the research communities are currently focused on hybrid electrical aircrafts where different strategies have been proposed [1]-[5] by various researchers to use integrated conventional fuel and energy storage systems (either battery or SMES) in order to achieve the goals. It includes the contribution of both conventional jet engines technology and

Revised Manuscript Received on December 02, 2019

* Correspondence Author

Abhinav Kumar*, School of Mechanical Engineering, Lovely Professional University, Phagwara, India. Email: drabhinav@ieee.org

Ashish Agrawal, Department of Mechanical Engineering, Madanapalle Institute of Technology and Science, Madanapalle, India. Email: drashishagrawal@mits.ac.in

novel electric aircraft systems where electrical power can be used in take-off, climbing and landing of the aircraft as it only contributes to $25 \%$ of the total power consumption [6]. For this to achieve, various battery operated systems have been evolved during past few years such as Lead acid, Nickel metal hydride batteries and Lithium ion batteries. However, these batteries have limited storage capacities per unit weight/surface area (Figure 1 and Figure 2) and therefore for larger power capacities the overall weight and surface area will increase which directly affects the lift force.

The advanced electric aircraft like N3-X is equipped with HTS motors, SMES, superconducting generators and fault current limiters. All these superconducting systems are having an arrangement of multi-turns pancake coils [7]-[9]. For the transportation of electric current, HTS cables are proposed by NASA and AFRL [10]. Due to the presence of time-varying magnetic fields, it is obvious that all the superconducting systems experiences AC losses which are generally not required. AC losses are very sensitive to frequency and magnitude of current and found to increase with both frequency and current. Various other methods have been employed to control the AC losses at constant frequency and current [11][12].

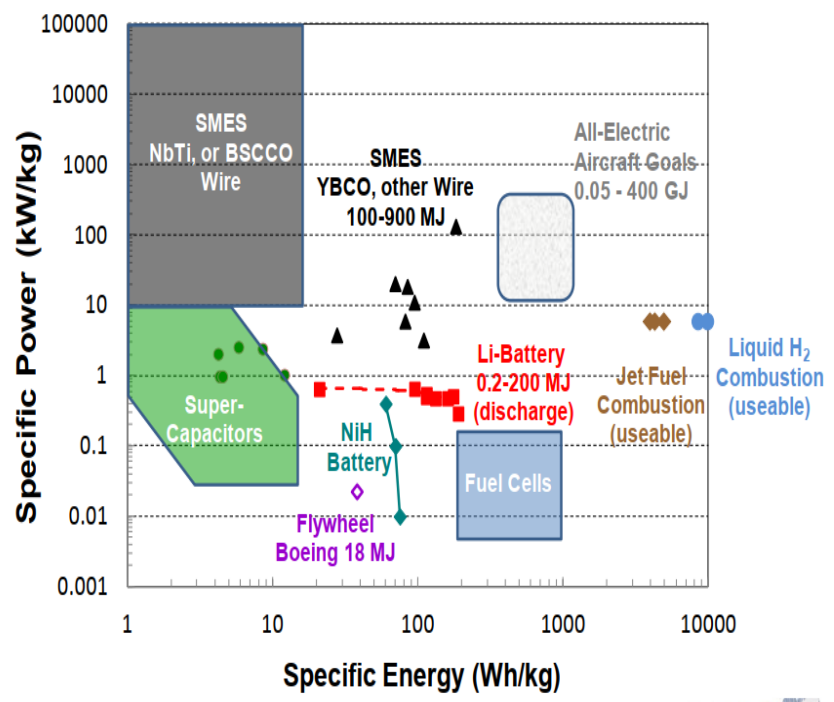

Fig. 1. Specific Power Vs Specific Energy comparison for various Energy storage systems [10] 
Effect of Substrate Layer Thickness on the AC Losses in Stacked Superconducting Pancake Coils using Direct H-formulations

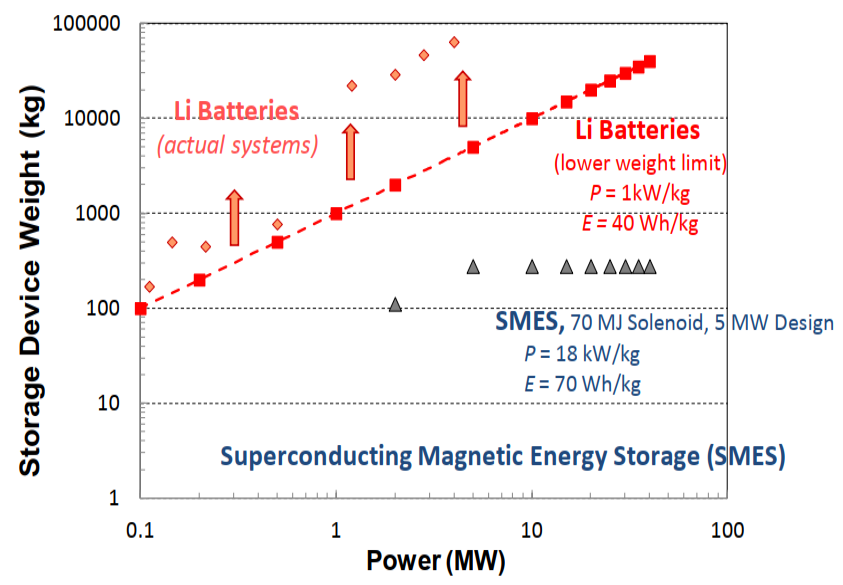

Fig. 2 Storage device weight Vs Power comparison for various Energy Storage Systems [10]

In this work, the effect of substrate layer thickness on AC losses has been examined. Superconducting tape (SCS 12050) of width $12 \mathrm{~mm}$ has been considered for the analysis whose parameters are tabulated in Table I. AC loss analysis has been performed using Comsol software package at $50 \mathrm{~Hz}$ frequency.

\section{COMPUTATIONAL MODEL}

A 2D computational domain has been developed using Comsol consisting air and superconducting domains as shown in Fig. 3. The resistivity of the superconducting tape has been modelled using E-J power law relationship (3) and the resistivity of air domain is modelled using $E=\rho J$. $\mathrm{H}$-formulations with E-J power law have been used in [13][14] where authors have employed edge elements for the discritization. Here, same methodology has been employed in order to evaluate the effect of the substrate thickness on the AC losses. H-formulations is a simple and accurate technique and results can be achieved faster than other related schemes A-V [15][16] and T- $\Omega$ [17][18] formulations. The PDEs used for the modelling of the stacked superconducting tapes are given as follows:

$\nabla \times \rho \nabla \times H=-\mu \frac{\partial H}{\partial t} \quad$ within domain

$H=H_{\text {self }}+H_{\text {ext }}$ on boundaries

$\rho_{H T S}=\frac{E_{c}}{J_{c}}\left|\frac{J}{J_{c}}\right|^{n-1}$

Instantaneous AC losses, $\xi=\int_{\text {Insidedomain }} E . J d x d y$

Kim model (5) [19] has been employed to consider the critical current density dependence on the local magnetic field, where $\mathrm{B}_{0}=0.04265 \mathrm{~T}, \mathrm{Jc}(77 \mathrm{~K}, \mathrm{SF})=2.75 \times 10^{10} \mathrm{~A} / \mathrm{m}^{2}, \mathrm{k}=0.29515$, $\alpha=0.7$ and $B_{I I}$ and $B_{\perp}$ represents the magnetic field density in parallel and perpendicular direction with respect to tape's surface [19].

$$
J_{c}(B)=\frac{J_{c}(77 K, S F)}{\left(1+\frac{\sqrt{k^{2}\left|B_{I I}\right|^{2}+\left|B_{\perp}\right|^{2}}}{B_{0}}\right)^{\alpha}}
$$

$\operatorname{normB}=\sqrt{B_{I I}^{2}+B_{\perp}^{2}}$

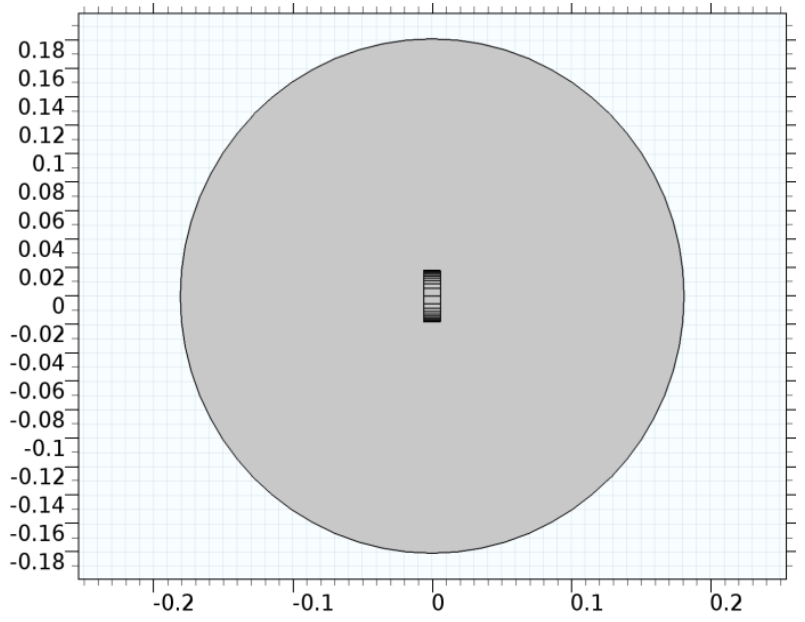

Fig. 3. Homogenized computational domain

Table- I: Design Parameters of the pancake coil

\begin{tabular}{|l|l|}
\hline Description & Value \\
\hline Maximum Current & $270 \mathrm{~A}$ \\
\hline Index, $\mathrm{n}$ & 30 \\
\hline Total number of turns & 108 \\
\hline $\mathrm{Cu}$ cover height (each side) & $20 \mathrm{e}-6 \mathrm{~m}$ \\
\hline Air gap and insulator height & $2 \mathrm{e}-4 \mathrm{~m}$ \\
\hline Ag cover height & $4 \mathrm{e}-6 \mathrm{~m}$ \\
\hline Substrate height & $30,50,70,90 \mu \mathrm{m}$ \\
\hline HTS layer height & $1 \mathrm{e}-6 \mathrm{~m}$ \\
\hline Tape height & $1 \mathrm{e}-4 \mathrm{~m}$ \\
\hline Tape width & $12 \mathrm{e}-3 \mathrm{~m}$ \\
\hline Resistivity of Air & $1 \mathrm{~m} * \mathrm{~V} / \mathrm{A}$ \\
\hline Resistivity of Ag & $2.7 \mathrm{e}-9 \mathrm{~m} * \mathrm{~V} / \mathrm{A}$ \\
\hline Resistivity of Cu & $1.97 \mathrm{e}-9 \mathrm{~m} * \mathrm{~V} / \mathrm{A}$ \\
\hline Resistivity of substrate & $1.25 \mathrm{e}-9 \mathrm{~m} * \mathrm{~V} / \mathrm{A}$ \\
\hline Frequency of transport current & $50 \mathrm{~Hz}$ \\
\hline Critical Current Density, Jc & $2.75 \mathrm{E} 11 \mathrm{~A} / \mathrm{m}^{2}$ \\
\hline
\end{tabular}

\section{RESULTS AND DISCUSSIONS}

\section{A. Magnetic flux and current density distribution}

Fig. 4 and Fig. 5 shows the $\mathrm{J} / \mathrm{Jc}$ and normB distribution for different substrate thicknesses (30 to $90 \mu \mathrm{m}$ ) at $50 \mathrm{~Hz}$ sinusoidal frequency respectively. It can be noticed that the $\mathrm{J} / \mathrm{Jc}$ ratio is found to reduce with substrate thickness. Same trend is repeated for magnetic flux density distribution which implies less dense magnetic flux is available at the core. 


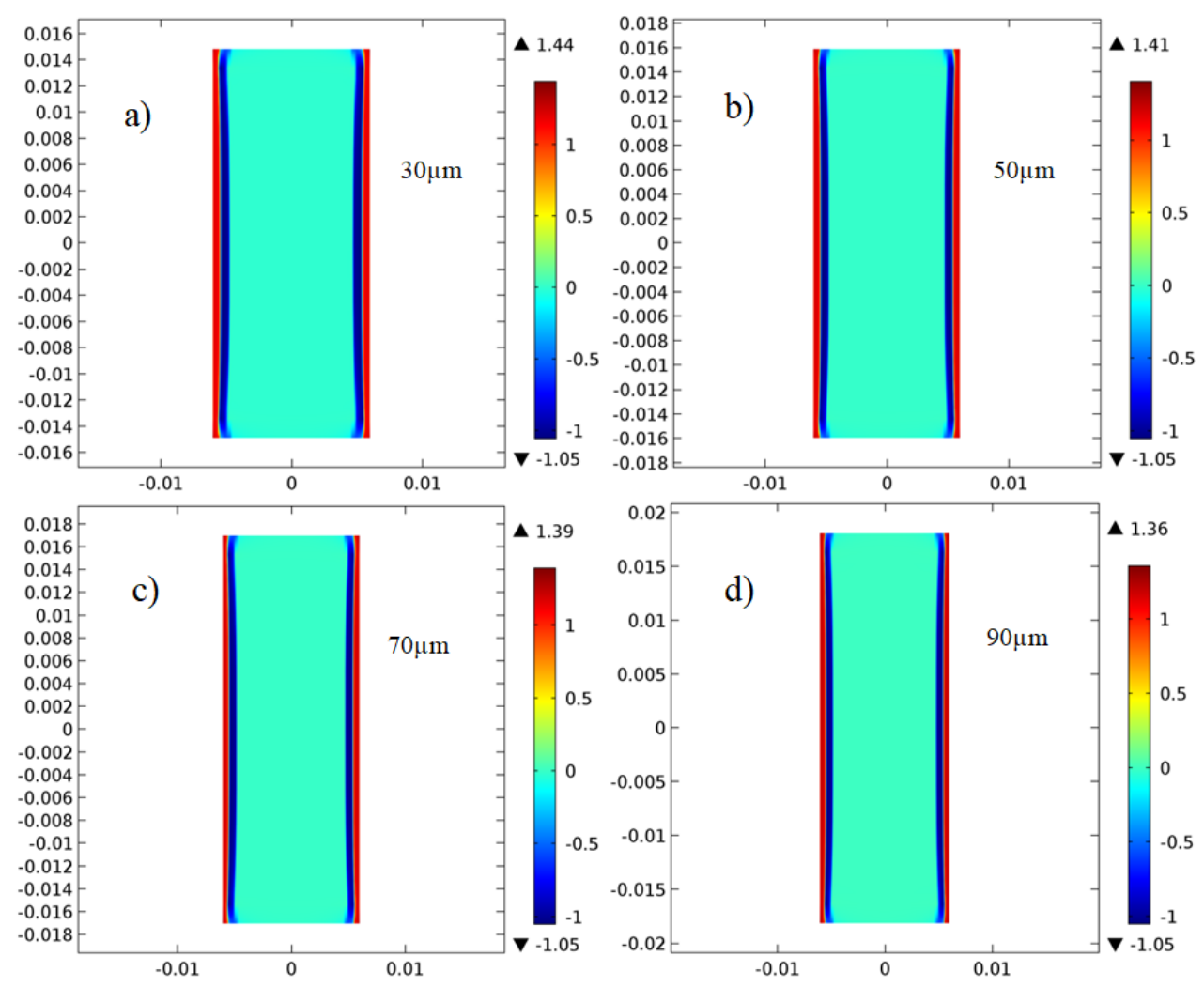

Fig. 4. J/Jc distribution for different substrate thicknesses
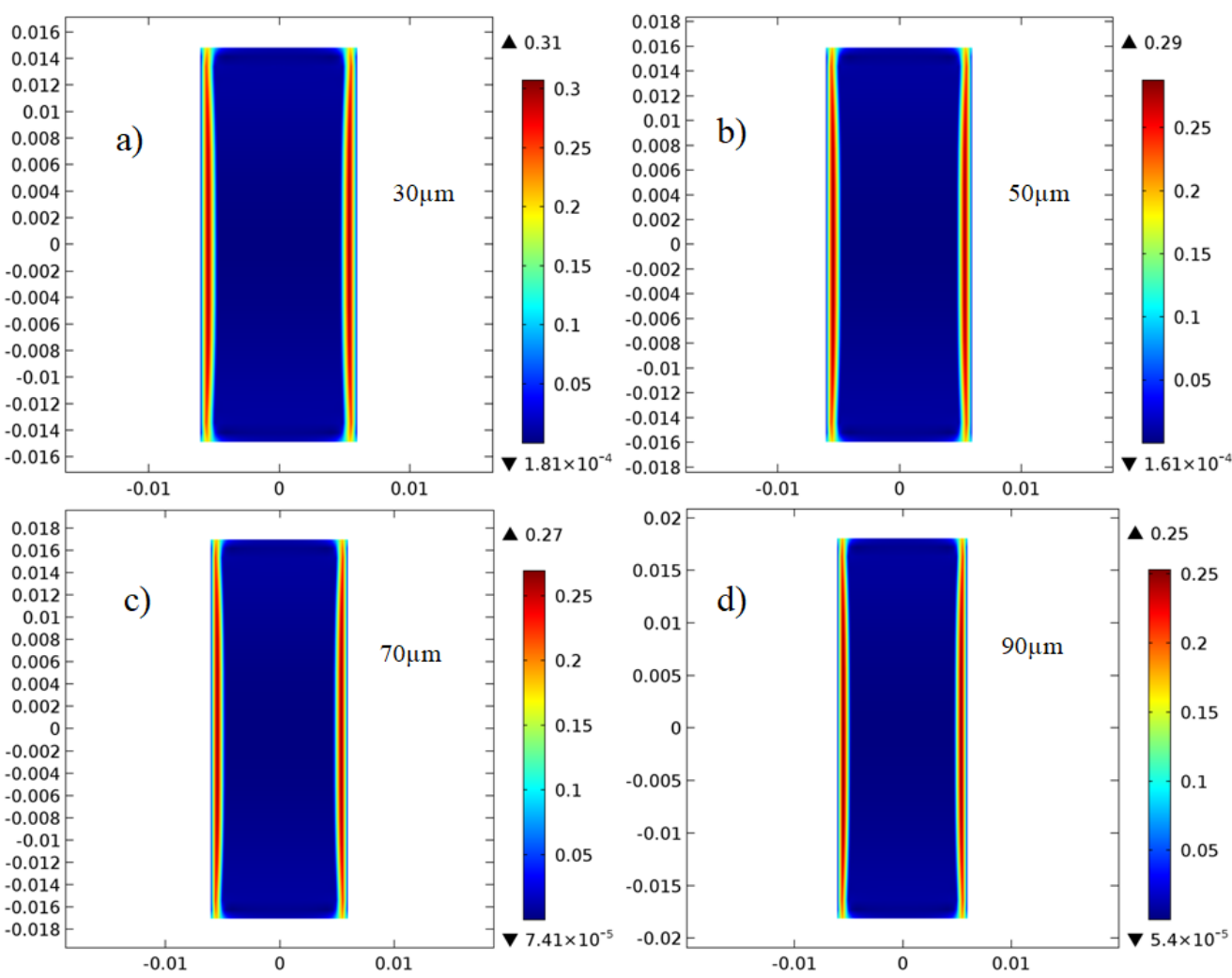

Fig. 5. Magnetic flux density distribution for different substrate thicknesses 


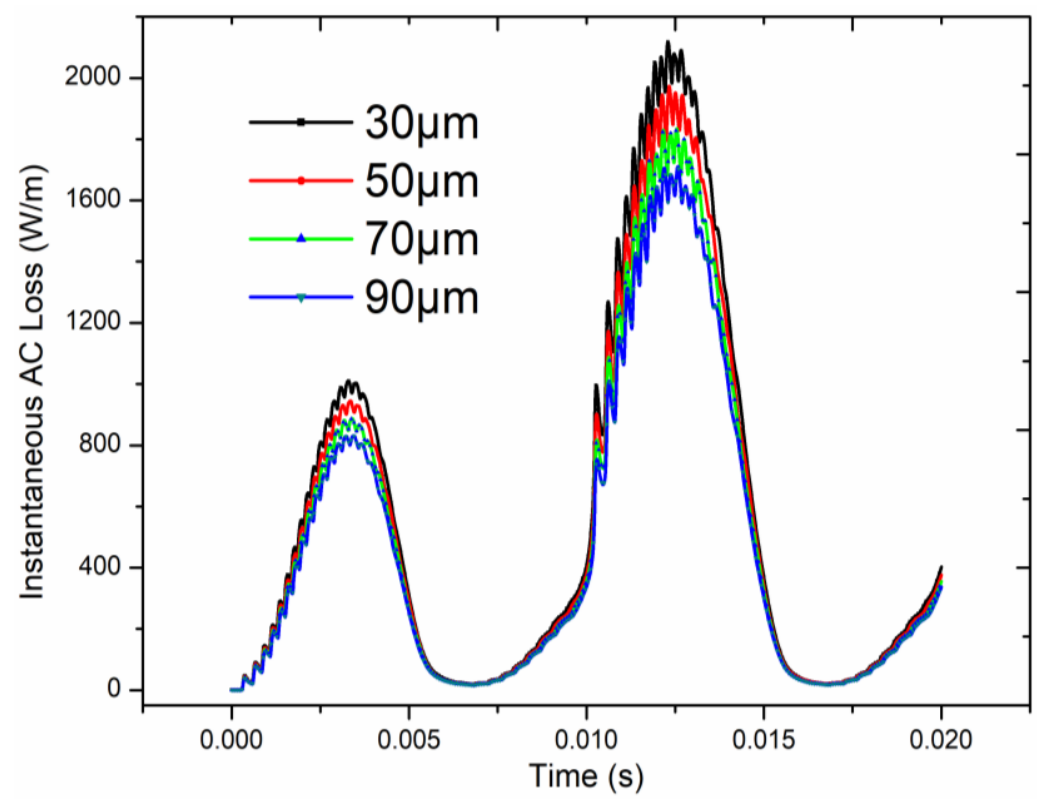

Fig. 6. Instantaneous AC Losses (W/m) for different substrate thicknesses

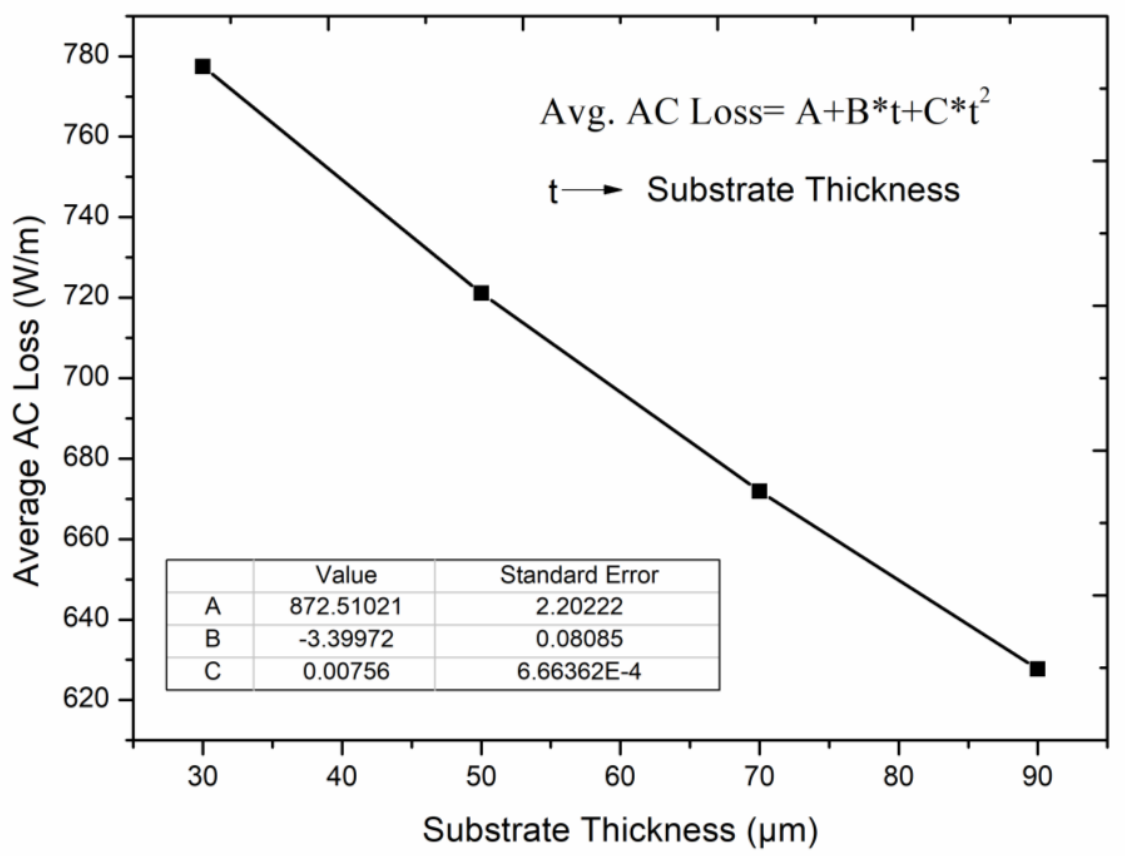

Fig. 7. Average AC losses vs. substrate thickness

\section{B. AC Losses}

Fig. 6 and Fig. 7 shows the instantaneous and average AC loss variation for various substrate thicknesses. It can be seen from Fig. 6 that the losses are found to decrease with increase in substrate's thickness. Fig. 7 indicates the average losses which found to decrease with thickness parabolically. To estimate the average losses within 30 to $90 \mu \mathrm{m}$, a correlation has been made whose parameters are given in Fig. 7 .

\section{CONCLUSION}

Lower AC losses are always desired in the superconducting applications which are generally appears for few fraction of seconds in the initial stages when current starts approaching to its steady state magnitude.
In the present study, the effect of substrate material thickness on the AC losses have been examined and results showed that there is a significant reduction in the AC losses with increase in thickness however at the cost of magnetic flux density which is also found to decline with thickness. This implies compromise has to make while choosing the substrate material thickness which further depends upon the type of applications (motors, energy storage, transformers, generators, fault current limiters etc.) where such superconducting pancake coils are to be used. 


\section{REFERENCES}

1. J. Sliwinski, A. Gardi, M. Marino, and R. Sabatini, "Hybrid-electric propulsion integration in unmanned aircraft," Energy, vol. 140, pp. 1407-1416, 2017.

2. C. E. D. Riboldi, "An optimal approach to the preliminary design of small hybrid-electric aircraft,” Aerosp. Sci. Technol., vol. 81, pp. 14-31, 2018.

3. C. Pornet and A. T. Isikveren, "Conceptual design of hybrid-electric transport aircraft,” Prog. Aerosp. Sci., vol. 79, pp. 114-135, 2015.

4. E. Frosina, C. Caputo, G. Marinaro, A. Senatore, C. Pascarella, and G. Di Lorenzo, "Modelling of a Hybrid-Electric Light Aircraft," Energy Procedia, vol. 126, pp. 1155-1162, 2017.

5. P. C. Vratny and M. Hornung, "Sizing Considerations of an Electric Ducted Fan for Hybrid Energy Aircraft," Transp. Res. Procedia, vol. 29 pp. 410-426, 2018.

6. S. Ma, S. Wang, C. Zhang, and S. Zhang, "A method to improve the efficiency of an electric aircraft propulsion system," Energy, vol. 140, pp. 436-443, 2017.

7. A. Kumar, A. Agrawal, and J. M. L. Jeyan, "A Numerical Model Comprising the Effect of Number of Turns on AC Losses in 2G HTS Coated Conductor at 77K using $\mathrm{H}$-formulations," in 2019 IEEE 2nd International Conference on Power and Energy Applications (ICPEA), 2019, pp. $115-118$.

8. A. Kumar and R. Kaur, "Electromagnetic analysis of $1 \mathrm{MJ}$ class of high temperature superconducting magnetic energy storage (SMES) coil to be used in power applications," vol. 050003, p. 050003, 2018.

9. A. Kumar, J. V. M. L. Jeyan, and A. Agarwal, "Numerical analysis on $10 \mathrm{MJ}$ solenoidal high temperature superconducting magnetic energy storage system to evaluate magnetic flux and Lorentz force distribution," Phys. C Supercond. its Appl., vol. 558, pp. 17-24, 2019.

10. T. J. Haugan, "Development of Superconducting and Cryogenic Power Systems and Impact for Aircraft Propulsion," Air Force Res. Lab., no. April, 2015.

11. N. Amemiya, T. Tsukamoto, M. Nii, T. Komeda, T. Nakamura, and Z. Jiang, "Alternating current loss characteristics of a Roebel cable consisting of coated conductors and a three-dimensional structure," Supercond. Sci. Technol., vol. 27, no. 3, p. 35007, 2014.

12. N. J. Long, R. A. Badcock, K. Hamilton, A. Wright, Z. Jiang, and L. S. Lakshmi, "Development of YBCO Roebel cables for high current transport and low AC loss applications," vol. 022021, no. 11, pp. 1-9, 2010.

13. Brambilla, F. Grilli, and L. Martini, "Development of an edge-element model for AC loss computation of high-temperature superconductors," vol. 16, 2007.

14. Z. Hong, A. M. Campbell, and T. A. Coombs, "Numerical solution of critical state in superconductivity by finite element software," Supercond. Sci. Technol., vol. 19, no. 12, p. 1246, 2006.

15. F. Gömöry, M. Vojenčiak, E. Pardo, and J. Šouc, "Magnetic flux penetration and AC loss in a composite superconducting wire with ferromagnetic parts," Supercond. Sci. Technol., vol. 22, no. 3, p. 34017, 2009.

16. A. Stenvall and T. Tarhasaari, "An eddy current vector potential formulation for estimating hysteresis losses of superconductors with FEM," Supercond. Sci. Technol., vol. 23, no. 12, p. 125013, 2010.

17. N. Amemiya, S. Murasawa, N. Banno, and K. Miyamoto, "Numerical modelings of superconducting wires for AC loss calculations," Phys. C Supercond., vol. 310, no. 1-4, pp. 16-29, 1998.

18. N. Enomoto and N. Amemiya, "Electromagnetic field analysis of rectangular high Tc superconductor with large aspect ratio," Phys. C Supercond., vol. 412, pp. 1050-1055, 2004.

19. K. P. Thakur, A. Raj, E. H. Brandt, J. Kvitkovic, and S. V Pamidi, "Frequency-dependent critical current and transport ac loss of superconductor strip and Roebel cable,” vol. 065024, 2011.

\section{AUTHORS PROFILE}

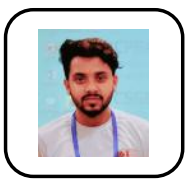

Abhinav Kumar is pursuing his Doctorate in Mechanical Engineering from Lovely Professional University, Punjab, India. He has completed his master's in thermal engineering specialization from Lovely Professional University in 2014 and bachelor's in Mechanical Engineering from Punjab Technical University in 2011. He has published many SCI and Scopus indexed articles in various journals and has attended many international conferences. His area of research is vast including superconducting magnetic energy storage systems, superconducting fault current limiters, high temperature superconducting cable design \& cooling, cryogenic fluid properties, nozzle cooling, computational fluid dynamics and solar energy storage systems. $\mathrm{He}$ is one of the reviewers for SCI and Scopus indexed journals and conferences. $\mathrm{He}$ has been awarded with University Academic Honor and University Honor Roll for his excellent academic records. He is holding Student IEEE and life membership of Indian Society of Heat and Mass Transfer (ISHMT).

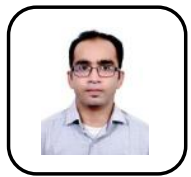

Dr. Ashish Agrawal is an Assistant Professor at the Department of Mechanical Engineering in Madanapalle Institute of Technology and Science in Madanapalle, Andhra Pradesh in India. He obtained his Masters and PhD from Indian Institute of Technology, Kanpur in 2010 and 2017 respectively. He graduated in Mechanical Engineering from Madhav Institute of Technology and Science in Gwalior, Madhya Pradesh in India. Prior joining to MITS Madanapalle, he has worked as an Assistant Professor at Lovely Professional University in Punjab. Broadly his research interests lie in computational and experimental heat transfer and fluid dynamics. More specifically, his research sears include heat transfer in rotary kilns, electronics cooling, superconducting magnetic energy storage system and vortex tube cooling system. 